\title{
Estados emocionais e relacionamento familiar de cuidadores de crianças com queimaduras
}

\author{
Adriano Valério dos Santos Azevêdo \\ Maria Aparecida Crepaldi
}

\section{RESUMO}

Este artigo buscou apresentar uma revisão integrativa de pesquisas empíricas referentes a estados emocionais e relacionamento familiar de cuidadores de crianças com queimaduras. Foram realizadas buscas de artigos empíricos e de revisão nas bases de dados Medline, Lilacs, Science Direct, Pubmed e Psyclnfo para selecionar os estudos publicados entre 1993 e 2015. Foram identificados 36 artigos da produção internacional que estão concentrados nas seguintes temáticas: ansiedade e transtorno de estresse pós-traumático, depressão, estresse parental e relacionamento familiar. Os resultados indicaram a presença de ansiedade decorrente de preocupações com a evolução clínica da criança. Verificou-se a ocorrência de estresse póstraumático, principalmente nos pais que presenciaram o acidente doméstico que provocou queimaduras. Níveis de depressão estão relacionados com a idade jovem do cuidador, e a presença de estresse parental é proveniente do processo de tratamento de queimaduras do filho. Foram identificadas mudanças estruturais decorrentes do processo de reinserção da criança no contexto familiar, assim, no relacionamento familiar existe uma fase de conflitos e de meIhorias nas relações entre irmãos. Isso indica a necessidade de direcionar atenção integral à criança e sua família na unidade de queimados por meio de acompanhamento no período de hospitalização e durante a fase de retorno ao contexto familiar.

Palavras-chave: cuidadores familiares; queimaduras; criança hospitalizada.

\section{ABSTRACT}

\section{Emotional states and family relationship of caregivers of children with burns}

The aim of this article was to present an integrative review of empirical research on the emotional states and family relationships of caregivers of children with burns. Empirical and review articles were searched on Medline, Lilacs, Science Direct, PubMed, and PsycINFO databases to select studies published between 1993 and 2015. Thirty-six international articles focusing on the following topics were found: anxiety and post-traumatic stress disorder, depression, parental stress, and family relationships. Results indicated the presence of anxiety due to concerns regarding the clinical progress of the child. The occurrence of post-traumatic stress was also found, mainly in parents who witnessed the domestic accident that caused the burns. Depression levels are related to young age of caregivers, while the presence of parental stress arises from the process of treating the child's burns. Structural changes resulting from the process of reintegration of the child into the family context were identified. There seems to be a phase of conflicts and improvement in sibling relations within the family relationship. These results show that it is necessary to dedicate full attention to the child and their family in the burns unit by monitoring the patient during hospitalization and as they return to the family context.

Keywords: family caregivers; burns; hospitalized child.

As lesões provocadas por queimaduras representam um evento traumático para a criança e sua família, ao considerar as implicações nos aspectos psicológicos de todos os envolvidos (Bakker \& Walstra, 2013; De Souza, 2010; Landolt, Buehlmann, Maag, \& Schiestl, 2009), devido às recordações do acidente, à necessidade de hospitalização prolongada e às mudanças na rotina que ocasionam sofrimento. Além disso, oferecer cuidados a uma criança com queimaduras representa uma tarefa difícil para os pais, devido às variáveis do contexto hospitalar, tais

\section{Sobre os Autores}

A. V. S. A. orcid.org/000-0003-0238-3423 Universidade Tuiuti do Paraná (UTP) - Curitiba, PR

adrianoazevedopsi@yahoo.com

M. A. C.

orcid.org/0000-0002-5892-7330

Universidade Federal de Santa Catarina (UFSC) - Florianópolis, $\mathrm{SC}$

crepg@gmail.com

\section{Direitos Autorais}

Este é um artigo de acesso aberto e pode ser reproduzido livremente, distribuído,

transmitido ou modificado, por qualquer pessoa desde que usado sem fins comerciais. 0 trabalho é disponibilizado sob a licença Creative Commons CCBY-NC.

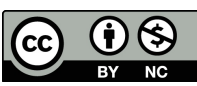




\section{H. INTERACÃO EM F PSICOLOGIA}

como ambiente fechado e regras institucionais, que de alguma maneira potencializam reações emocionais em virtude do processo de tratamento.

Queimaduras estão associadas ao fenômeno da dor para o qual existem diferentes intervenções farmacológicas (analgesia) e não farmacológicas (distração) visando o manejo adequado (Martin-Herz, Thurber, \& Patterson, 2000). Dessa forma, a experiência de situação de queimadura, as sensações dolorosas e o medo são compartilhados pela díade criança-família. Tecnologias avançadas são utilizadas para o tratamento de queimaduras, o que inclui cuidados intensivos de limpeza, controle de infecção e realização de cirurgias, para que isso facilite a sobrevivência de crianças (Sheridan, 2002). E mesmo diante desses avanços tecnológicos, a inserção numa unidade de queimados ocasiona nos acompanhantes das crianças a ansiedade pela busca de informações (Bicho \& Pires, 2002), o que provavelmente repercute na alternância entre momentos de incerteza e preocupações que envolvem todos os integrantes da família.

$\mathrm{Na}$ unidade de queimados para cuidados de crianças, a hospitalização e os tratamentos ocasionam o surgimento de reações emocionais e mudanças na dinâmica familiar (McGarry et al., 2013), aspectos que se refletem em preocupações com a evolução clínica, as sequelas das queimaduras, que inclui cicatrizes e limitações físicas, e o retorno da criança ao ambiente familiar e às atividades sociais. Nesse sentido, as alterações nos estados emocionais e no relacionamento familiar são provenientes dessa vivência marcada por momentos de tensão e de mudanças contínuas, de maneira que a identificação desses aspectos permite sistematizar pesquisas para fins de reflexão crítica e desenvolvimento de aplicações práticas de projetos que visam minimizar os efeitos provocados durante e após o período de hospitalização.

Investigar os estudos referentes aos cuidadores dessas crianças possibilita sintetizar o panorama da produção científica realizada ao longo dos anos para que se torne possível apresentar propostas de avaliação e intervenção psicológica. A busca sistemática das pesquisas permite identificar as variáveis que foram analisadas, os tipos de métodos e instrumentos utilizados para permitir avanços na delimitação de novos estudos. Considerando o exposto, este artigo tem por objetivo apresentar uma revisão integrativa de pesquisas empíricas referentes a estados emocionais e relacionamento familiar de cuidadores de crianças hospitalizadas com queimaduras. Os pesquisadores que realizaram essa revisão integrativa têm experiência na área de avaliação e intervenção psicológica hospitalar pediátrica, especificamente na área de queimaduras pediátricas, assim, a realização deste estudo foi motivada pelo interesse de conhecer evidências ci-

\section{Adriano Valério dos Santos Azevêdo e Maria Aparecida Crepaldi}

entíficas de pesquisas para aprimorar práticas de intervenção e pesquisa.

\section{MÉTODO}

Utilizou-se o delineamento de revisão integrativa, o que representa um tipo de pesquisa bibliográfica que investiga a produção científica de um objeto de estudo para construir temáticas e agrupar/integrar as pesquisas encontradas e, por meio dos resultados obtidos, apresentar uma síntese das evidências e destacar propostas de pesquisas e intervenção (Mendes, Silveira, \& Galvão, 2008). Dessa forma, a revisão integrativa contribui para o desenvolvimento de novas pesquisas e auxilia ações técnicas referentes às intervenções.

O estabelecimento de critérios auxiliou a sistematizar o processo de revisão que foi realizado por meio de consultas nas bases de dados Bireme - Medline, Lilacs, Science Direct, Pubmed e Psyclnfo. Foram selecionadas as pesquisas publicadas no período de 2010-2015, mas foram encontrados poucos estudos, assim, para a análise de publicações ao longo do tempo, buscou-se identificar estudos empíricos e de revisão publicados entre janeiro de 1993 até dezembro de 2015, por meio das palavras-chave: And ${ }^{*}$ queimadura, ${ }^{*}$ criança, *acompanhante, *familiares, Or *burn, *child, *family. Utilizouse a recomendação internacional - Preferred Reporting Items for Systematic Reviews and Meta-Analyses (PRISMA - disponível em: www.prisma-statement.org) - no processo de identificação, seleção e elegibilidade dos artigos com o propósito de atender critérios de qualidade.

\section{CRITÉRIOS DE INCLUSÃO E EXCLUSÃO}

Foram incluídos artigos que se referiam aos relatos de pesquisas empíricas e de revisão sobre familiares cuidadores de crianças com queimaduras, especificamente, investigações sobre estados emocionais e relacionamento familiar, durante e após o período de hospitalização. Foram excluídas as dissertações e teses; artigos mistos que se referiam aos acompanhantes de adultos e crianças; estudos de caso; estudos sobre programas de prevenção de queimaduras; estudos epidemiológicos; pesquisas que solicitavam que os pais avaliassem o comportamento das crianças; publicações relacionadas à construção e validação de instrumentos; e outras que buscavam avaliar a satisfação dos pais sobre os serviços oferecidos no hospital. Os artigos que atendiam os critérios de inclusão foram selecionados, inicialmente, para leitura do resumo e, em seguida, para análise integral do texto para identificar a temática de estudo, o método e os principais resultados, o que auxiliou o agrupamento de temáticas. 


\section{W INERACÄOEM ET PSICOLOGIA}

\section{RESULTADOS}

A Figura 1 apresenta o fluxograma do trabalho, destacando-se que no processo de identificação foram aplicados os critérios de inclusão, em seguida foram excluídos artigos repetidos e estudos sobre crianças e aqueles referentes à epidemiologia, testagem de procedimentos e avaliação de intervenção relacionados à área da medicina e enfermagem. $\mathrm{Na}$ próxima etapa foram considerados elegíveis os artigos que apresentavam no título o termo referente a pais e acompanhantes, e após a leitura do objetivo foram incluídos estudos que investigaram temáticas referentes a estados emocionais e relacionamento familiar. Os estudos são provenientes dos seguintes países: Estados Unidos da América,
Adriano Valério dos Santos Azevêdo e Maria Aparecida Crepaldi

Holanda, África do Sul, Inglaterra, Marrocos e Austrália. Foram analisados os estudos referentes a estados emocionais de ansiedade, depressão, estresse, estresse pós-traumático e pesquisas sobre o relacionamento familiar.

A seguir serão apresentadas as sínteses das pesquisas que foram incluídas nesta revisão integrativa.

\section{ANSIEDADE E TRANSTORNO DE ESTRESSE PÓS-TRAUMÁTI- CO}

Um estudo qualitativo realizado por Bicho e Pires (2002) que entrevistou quatro mães mostrou que o processo de hospitalização para o tratamento de queimaduras é gerador de mudanças psicológicas nos pais, pois eles apresentam ansie-

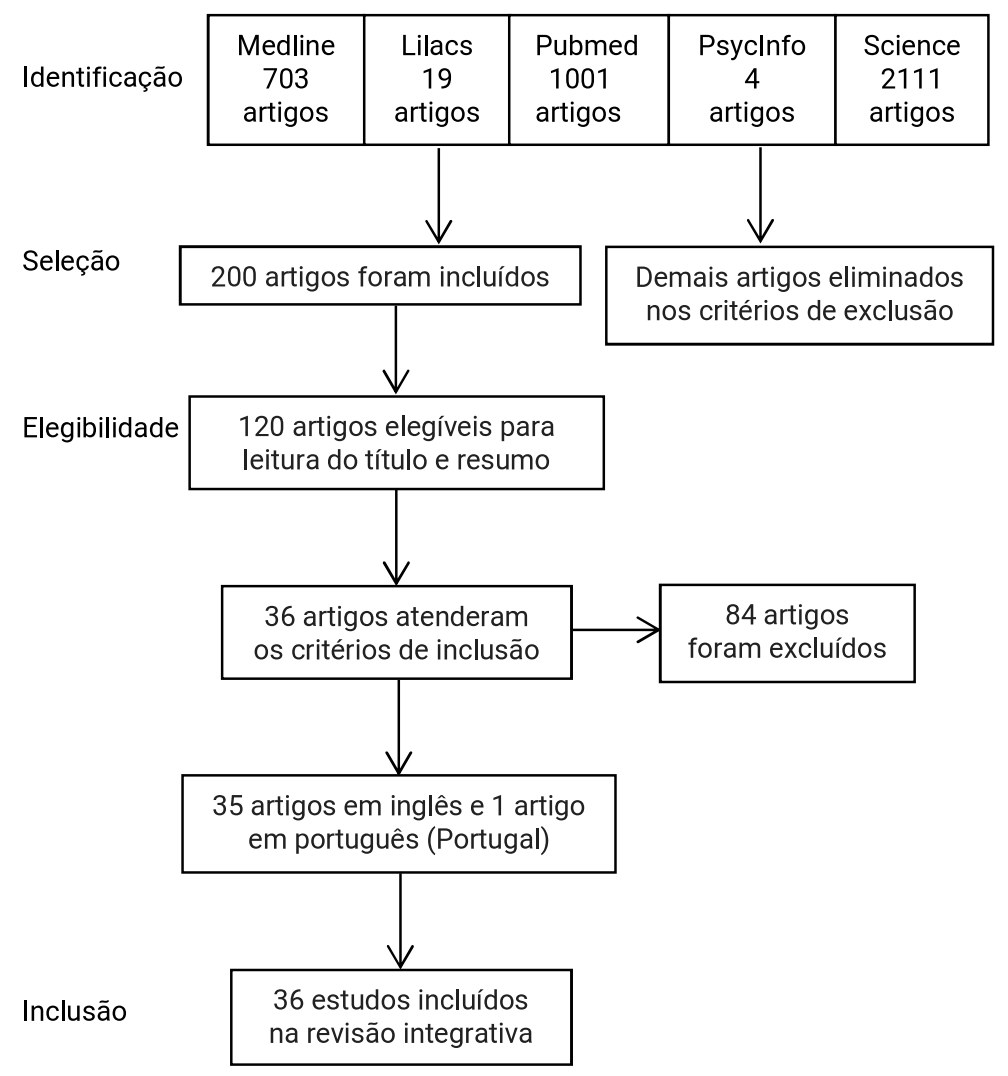

Figura 1. Fluxograma do processo de busca e seleção dos artigos.

dade pela busca de informações sobre a condição clínica da criança e se preocupam em reduzir o sofrimento emocional do filho. Para minimizar o sofrimento da criança, os pais oferecem apoio emocional e ficam constantemente ao lado da criança, pelo fato de que vivenciam a angústia diante do quadro clínico do filho e da dependência dos profissionais de saúde. Assim, percebe-se que nesse apoio existe o desenvol- vimento da empatia na relação entre pais e criança, o que contribui para minimizar o sofrimento compartilhado por ambos e, dessa forma, auxiliar o enfrentamento da hospitalização.

Numa amostra de 97 participantes, identificou-se que nos primeiros dias de hospitalização as preocupações dos pais envolveram aspectos relacionados à saúde, à dor e ao futuro 
da criança, especificamente referentes à cirurgia de enxerto da pele, em que também recebiam apoio emocional da família, dos amigos e da equipe de saúde (Thompson, Boyle, Teel, Wambach, \& Cramer, 1999). Reações emocionais de pânico e alternância entre sentimentos de culpa e raiva foram verificados nas entrevistas realizadas com 21 pais (14 mães e 7 pais), de maneira específica, naqueles que presenciaram o evento que provocou a queimadura na criança (McGarry et al., 2015). Em síntese, ansiedade e culpa foram vivenciadas desde o primeiro momento da ocorrência do acidente e, dessa forma, possivelmente existe uma tendência de agravamento de acordo com a situação clínica da criança e a experiência vivenciada pelos pais, aspectos a serem considerados numa unidade de queimados.

Kent, King, e Cochrane (2000), em uma pesquisa, investigaram a ansiedade de 40 mães de crianças com queimaduras comparando com a ansiedade de um grupo de 40 mães de crianças com fraturas, no início da hospitalização e após seis meses, o que ocorreu por meio da aplicação da Hospital Anxiety and Depression Scale (HADS). A ansiedade do primeiro grupo foi superior (9.3) quando comparada ao segundo grupo (6.0). Verifica-se que isso indica a necessidade de investigação e intervenção para fins de promover acolhimento ao familiar e à criança no momento da hospitalização. Em outro estudo que utilizou a HADS com 16 cuidadores de crianças com queimaduras, verificou-se que 11 pais apresentaram ansiedade em excesso, 6 pais com ansiedade moderada e 4 com ansiedade severa (Phillips \& Rumsey, 2008).

Na pesquisa de McGarry et al. (2013), verificou-se que, na primeira semana de hospitalização, 63 pais de crianças com queimaduras apresentaram Sintomas de Transtorno de Estresse Pós-Traumático $(M=36,22 ; p=0,02)$, e as médias foram maiores $(M=38,88 ; p=0,02)$ naqueles pais que presenciaram o evento da queimadura, vivenciaram sentimento de desamparo e recordaram experiências que ocorreram em algum momento da vida, o que repercutiu em distress, que se refere ao estresse crônico proveniente da baixa capacidade para superar as situações de estresse, e baixos níveis de resiliência que foram identificados por meio de entrevista (McGarry et al., 2013). Verifica-se que o fato de presenciar o acidente que provocou queimadura potencializa reações de estresse, assim, é possível destacar que se trata de um impacto emocional gerador de repercussões psicológicas, algo que necessita de atenção por parte de todos os envolvidos na unidade hospitalar.

Fukunishi (1998) identificou numa amostra de 16 mães nos primeiros meses após o evento da queimadura que apenas três mulheres apresentaram Sintomas de Transtorno de Estresse Pós-Traumático e, após quatro anos, não foram identificados esses sintomas, o que ocorreu pelo fato de que as famílias receberam apoio emocional e estabeleceram bo- as relações no contexto familiar. No estudo de Hall et al. (2006), após três meses da ocorrência de queimaduras, ambos os pais das crianças desenvolveram esses sintomas ( $n=62 / 47 \%$ ), os quais foram relacionados com os conflitos vivenciados na relação pais e criança, especificamente, os sintomas de TEPT da criança mobilizaram reações psicológicas nos pais. Esses resultados indicam que no contexto de queimaduras pediátricas a qualidade das relações familiares e do apoio recebido apresentam repercussões nos estados emocionais da criança e do familiar cuidador, mas verificouse no primeiro estudo a ausência de sintomas de estresse pós-traumático após alguns anos, o que significa que o familiar cuidador conseguiu superar a situação referente à hospitalização.

Um estudo com 16 mães de crianças sem sequelas de queimaduras utilizou entrevista diagnóstica do DSM e foi verificado que essas mães não apresentaram sintomas de TEPT (Fukunishi, 1998). Outros autores (Rizzone, Stoddard, Murphy, \& Kruger, 1994) identificaram por meio de entrevista clínica diagnóstica que os pais de crianças queimadas sem sequelas apresentaram sintomas de TEPT no mês anterior à hospitalização da criança, mas numa amostra de 25 participantes, apenas quatro desenvolveram sintomas que caracterizavam TEPT. É notável a diferença do surgimento de reações emocionais nas famílias de crianças sem sequelas, assim, é importante investigar se essas famílias vivenciaram, no período anterior ao acidente que provocou queimaduras, sintomas de estresse pós-traumático por considerar que o desenvolvimento de intervenções para os casos mais graves representa uma estratégia de atenção à saúde integral.

Um estudo de revisão de pesquisas sobre Transtorno de Estresse Pós-Traumático (TEPT) em unidades intensivas para tratamento de queimaduras mostrou que, após a admissão da criança, há variações de $10,5 \%$ a $21 \%$ de ocorrência de sintomas de estresse traumático nos pais (Nelson \& Gold, 2012). Além disso, existem percentuais de sintomas que estão próximos de 84\%; as mulheres apresentaram os escores mais altos e existe uma relação entre sintomas de estresse da criança e dos pais, por exemplo, o estresse pós-traumático vivenciado pela criança é compartilhado com o familiar cuidador, este que desenvolve sintomas de TEPT.

\section{DEPRESSÃO}

No estudo realizado por Fukunish (1998) numa amostra de 16 mães de crianças sem sequelas, o escore obtido por meio da Escala Hamilton de Depressão foi de 14.2, o que foi considerado leve, e não foram encontradas correlações significativas entre depressão e sintomas de TEPT. Após quatro anos, o instrumento foi reaplicado e o escore 6.1 indicou ausência de sintomas de depressão. 


\section{-4* INTERACÃO EM LF PSICOLOGIA}

$\mathrm{Na}$ pesquisa realizada por LeDoux, Meyer, Blakeney, e Herndon (1998), o Inventário Beck de Depressão foi aplicado em 35 participantes, e o escore geral apresentou indicador abaixo de 15. Respectivamente, oito participantes apresentaram sintomas de depressão, e um participante depressão moderada. Outro estudo, realizado com 16 pais por meio da utilização da HADS, indicou que 4 foram classificados com depressão moderada, e alguns aspectos foram associados, por exemplo, idade jovem da mãe e pouca extroversão parental (Phillips \& Rumsey, 2008). A idade jovem da mãe e a falta de experiência no acompanhamento hospitalar de uma criança com queimaduras representam fatores que ocasionam sintomas depressivos, dessa forma, o acolhimento oferecido pela unidade hospitalar representa um diferencial para a proteção da saúde integral da criança e do familiar cuidador.

Uma pesquisa longitudinal realizada por Kent et al. (2000) acompanhou 40 mães de crianças com queimaduras após seis meses do período de hospitalização da criança e, por meio da utilização da HADS, comparou as médias com o grupo de mães de crianças com fraturas. Os resultados indicaram que o grupo de mães de crianças com queimaduras apresentou escores superiores de depressão (5.0) se comparado ao outro grupo (3.6). Rivlin e Faragher (2007) verificaram que no período de 3-14 anos após a ocorrência da queimadura da criança, 24 mães apresentaram maiores sintomas de depressão - cansaço, culpa, preocupações, tensões e humor deprimido - se comparado às mães de crianças com fraturas.

Num estudo com 28 mães por meio da aplicação da HADS e entrevista diagnóstica, verificou-se que 10 participantes apresentavam sintomas de depressão e foram considerados os seguintes fatores preditivos: severidade da queimadura, complicações ocorridas durante o período de hospitalização e características referentes ao nível socioeconômico (El Hamaoui, Yaalaoui, Chihabeddine, Boukind, \& Moussaoui, 2006). Famílias com nível socioeconômico baixo e em situação de vulnerabilidade social representam um grupo com predisposição para o surgimento de sintomas de depressão, o que provavelmente ocorre devido às dificuldades financeiras para os cuidados da criança pós-hospitalização. Relações entre o tamanho da queimadura e a depressão no primeiro ano não foram encontradas em outros estudos (Kent et al., 2000; Phillips \& Rumsey, 2008).

\section{ESTRESSE PARENTAL}

Reações de estresse agudo foram investigadas por meio da Escala de Impacto de Eventos (EIE) em 182 mães e 154 pais após o primeiro mês do evento da queimadura da criança (Bakker, Van Loey, Van der Heijden \& Van Son, 2012). Numa escala de $0-75$, a média das mães foi de 27.1 e a dos pais
Adriano Valério dos Santos Azevêdo e Maria Aparecida Crepaldi

17.5, em relação à percepção de sentimentos de culpa na escala de 0-4 (2.0 mães; 1.4 pais), e referente aos sentimentos de raiva na escala de $0-4$ (1.4 mães; 1.1 pais). É notável os escores elevados nas mães, o que indica que a figura materna vivencia estresse agudo após o período de hospitalização da criança. A Escala de Impacto de Eventos foi reaplicada em 48 mães respectivamente um ano após a queimadura cuja média foi de 12.50 , e onze anos depois da queimadura a média foi 6.18 , e embora tenha ocorrido um decréscimo nos escores, verificou-se que os sintomas de intrusão, que representam as alterações negativas na cognição e no humor referentes ao evento da queimadura, foram significativamente mais prevalentes do que os sintomas de evitação após um ano $\mathrm{t}(47)^{1 / 4} 6.62, \mathrm{p}<.001, \mathrm{Cl} 1 / 44.93-9.24$, e onze anos depois $\mathrm{t}(47)^{1} \frac{1}{4}$ 3.88, $\mathrm{p}<.001, \mathrm{Cl}^{1} / 4$ (Bakker, Van Loey, Maarten, Van Son, \& Van der Heijden, 2010). É importante destacar que as mães são as cuidadoras principais da criança, assim, a sobrecarga de cuidados é geradora de estresse, e mesmo considerando que a figura paterna oferece auxílio, as preocupações referentes ao processo de tratamento, reabilitação e ao futuro da criança são vivenciadas pela figura materna.

Outros pesquisadores identificaram a presença de estresse parental, mas a característica relacionada ao controle de conflitos não apresentou alterações (Liber, List, Van Loey, \& Kef, 2006; Pardo, Garcia, Marrero, \& Cia, 2008; Phillips \& Rumsey, 2008). Duas pesquisas identificaram que os altos níveis de estresse parental foram atribuídos às características da criança referentes às alterações no humor (Blakeney et al., 1998; Meyer et al., 1994),

Outras pesquisas identificaram que o estresse dos pais e as mudanças no funcionamento familiar foram atribuídos ao momento que a criança estava vivenciando, ou seja, o processo de tratamento de queimaduras (Landolt, Grubenmann, \& Meuli, 2002; Liber et al., 2006; Meyer et al., 1994; Rosenberg et al., 2007), pois não foi encontrada uma relação direta do estresse com a gravidade da queimadura (Blakeney et al., 1993b; Meyer et al., 1994). Verifica-se um consenso entre os autores de que o estresse parental é decorrente do momento atual da hospitalização da criança para tratamento de queimaduras, e, além disso, a qualidade das interações entre os integrantes minimiza ou intensifica os indicadores de estresse.

\section{RELACIONAMENTO FAMILIAR}

Um estudo qualitativo realizado com seis participantes destacou que os pais vivenciavam uma fase repleta de mudanças estruturais diante da reinserção da criança com queimaduras no contexto familiar e social e, ao se perceberem sozinhos, buscaram recursos para recuperar o bem-estar por 


\section{INTERACÃO EM * PSICOLOGIA}

meio do envolvimento entre os integrantes do núcleo familiar (Oster, Hensing, Löjdström, Sjöberg, \& Willebrand, 2014). Possivelmente, o envolvimento familiar por meio de fortalecimento de vínculos permitiu facilitar o enfrentamento dessa vivência e, com isso, auxiliou todos os integrantes da família.

A análise do relacionamento familiar indicou que em alguns ambientes domésticos ocorreu presença de problemas familiares nas interações que eram estabelecidas entre crianças e seus pais referentes às dificuldades de comunicação (Gorga et al., 1999); e em alguns casos que indicavam situação de vulnerabilidade social foram identificados maus-tratos e negligência (Meyer, Robert, Murphy, \& Blakeney, 2000). No estudo de LeDoux et al. (1998), verificou-se que os pais utilizaram a coesão familiar, que se refere às formas de envolvimento e de fortalecimento de vínculos entre seus integrantes, e o compartilhamento de aspectos religiosos (LeDoux et al., 1998; Rosenberg et al., 2007), o que representam maneiras de enfrentar as situações na fase pós-hospitalização da queimadura (LeDoux et al., 1998). Em outros casos, as famílias vivenciaram conflitos nas interações em longo prazo, e identificou-se a ocorrência de altas taxas de divórcios após o evento da queimadura (Blakeney et al., 1993a; Rivlin \& Faragher, 2007).

Estudos identificaram que crianças hospitalizadas com queimaduras são provenientes de famílias economicamente pouco favorecidas e que apresentam problemas socioeconômicos (Joseph, Adams, Goldfarb, \& Slater, 2002; Vendrusculo, Balieiro, Echevarría-Guanilo, Farina-Júnior, \& Rossi, 2010). E em outras pesquisas, verificou-se que existem ambientes familiares com suspeitas de negligência e abuso infantil (Peck \& Priolo-Kapel, 2002; Thombs, 2008). Uma pesquisa analisou a amostra de 150 crianças admitidas numa unidade de queimados, verificou-se que 16 crianças foram consideradas, de acordo com a avaliação da equipe de saúde, suspeitas de negligência e maus-tratos, e estes foram os principais fatores de risco: pais jovens, família monoparental, pouca idade da criança, queimaduras extensas, a demora da família para procurar atendimento, e relato contraditório sobre a ocorrência da queimadura que indicava violência ou negligência (Wibbenmeyer et al., 2014). Verifica-se que o tamanho da queimadura da criança representa um ponto para análise, assim, é possível verificar se existe alguma relação com a situação socioeconômica da família. A identificação desses aspectos permite que os profissionais de saúde desenvolvam habilidades para o estabelecimento de contatos com os pais da criança, por considerar que a escuta atenta auxilia a tomada de decisão, o que possibilita que os demais membros da equipe de saúde verifiquem as possibilidades de solicitação de profissionais do serviço social para análise da situação familiar.

No que se refere à relação familiar diante do retorno da criança à escola, verificou-se que a confiança dos pais em si
Adriano Valério dos Santos Azevêdo e Maria Aparecida Crepaldi

mesmo, na criança e na escola possibilitou o desenvolvimento de habilidades que auxiliaram no processo de adaptação (Horridge, Cohen, \& Gaskell, 2010). A receptividade da escola representou um aspecto que contribuiu para a reinserção da criança nas atividades escolares, o que permitiu auxiliar a relação criança-pais por meio do suporte com informações para o manejo das situações (Horridge et al., 2010). Outro aspecto incluiu as mudanças na família devido às despesas com o tratamento, ao considerar que famílias apresentaram alterações socioeconômicas após a ocorrência de queimaduras nos seus filhos, principalmente nos pais que tinham menor renda, pelo fato de que precisavam utilizar parte da renda para deslocamentos até o hospital (Kilburn \& Dheansa, 2014).

Pesquisas nas quais os acompanhantes foram respondentes indicaram dificuldades nas habilidades sociais de comunicação de crianças que têm um irmão/irmã com queimaduras moderadas ou graves (Mancuso, Bishop, Blakeney, Robert, \& Gaa, 2003). Verificou-se o envolvimento de irmãos nos cuidados das lesões do irmão ferido após dois anos da ocorrência da queimadura (Phillips, Fussell, \& Rumsey, 2007). Os irmãos, ao serem entrevistados, destacaram a importância de serem preparados para as visitas no centro de tratamento de queimados, pelo fato de que no primeiro momento vivenciaram sensação de choque (Phillips et al., 2007).

Identificou-se melhoria nas interações e no envolvimento de irmãos após alguns anos da ocorrência da queimadura, além disso, a maturidade referente aos cuidados e à proteção de um irmão com lesões provocadas por queimaduras (Mancuso et al., 2003). Após dois anos da ocorrência de queimaduras, o processo de ajustamento da criança ocorreu de maneira gradual por meio do brincar, do retorno à escola, o que posteriormente possibilitou desenvolver as relações com os irmãos (Lehna, 2010). Independentemente do número de irmãos, da idade, e do país em que moram, foi possível verificar a existência de contatos entre eles, o que ocorreu por meio de frequentes ligações telefônicas (Lehna, 2010).

É possível compreender que a investigação do relacionamento familiar possibilita a compreensão de diferentes aspectos, por exemplo, retorno ao ambiente familiar, à escola, interação com irmãos e envolvimento familiar. Esses aspectos caracterizam pontos específicos a serem estudados para que os resultados de pesquisas permitam a construção de projetos de intervenção na fase de internação e pós-hospitalização.

\section{DISCUSSÃO}

Ansiedade e Sintomas de Estresse Pós-Traumático são vivenciados pelos pais após a ocorrência de queimaduras e também na fase pós-hospitalização, o que ocorre por meio de 


\section{MI* INTERACÃO EM L PSICOLOGIA}

vivências de pânico e culpa. Na produção científica, verificouse que os sentimentos de culpa dos pais foram preditores para a utilização de estratégias de enfrentamento focalizadas no problema e na religião (Suurmond, Van Loey, Dokter, \& Essink-Bot, 2011).

A extensão da queimadura foi apresentada em alguns estudos, de maneira indireta, relacionada aos sintomas de TEPT de curto e longo prazo, pois ainda não existem evidências clínicas referentes à gravidade da queimadura e à ocorrência de estresse traumático. Por outro lado, a pesquisa de Hall et al. (2006) mostrou que as reações emocionais das crianças apresentaram repercussões no desenvolvimento de estresse traumático nos pais. De acordo com Kazak et al. (2006), eventos traumáticos, que incluem os acidentes domésticos em crianças que necessitam de hospitalização e tratamento intensivo, ocasionam reações de estresse, que são naturais e transitórias, a depender da maneira pela qual a criança vivencia a situação e enfrenta as mudanças referentes ao afastamento da escola e de familiares.

E no que se refere aos sintomas de estresse pós-traumático nos pais, é importante considerar que as mulheres, na grande maioria, acompanham seus filhos durante o período de hospitalização e nas fases seguintes de tratamento de reabilitação após alta hospitalar. As mulheres representam os membros da família mais pesquisados, e diante disso, esse resultado deveria alertar as equipes de saúde para que promovam uma atenção específica ao acompanhante que permanece com a criança, pois o familiar cuidador será mais afetado pela condição adversa que se configura o evento e consequente tratamento de queimaduras.

Os cuidados oferecidos pelos profissionais de saúde para a criança e família no período pós-hospitalização cumprem uma função educativa, o que permite realizar encaminhamentos para psicólogos e equipes de saúde responsáveis pela saúde da criança (Kazak et al., 2006). Diante das preocupações e angústias decorrentes do processo de hospitalização, o cuidado oferecido pela equipe de saúde possibilita auxiliar os pais no enfrentamento das situações (Oliveira, Fonseca, Leite, \& Santos, 2015), o que ocorrerá por meio do acolhimento e da atenção integral às necessidades da família com orientações aos pais sobre o processo de retorno da criança ao contexto familiar e escolar. Dessa forma, o acompanhamento por meio de uma equipe multiprofissional numa perspectiva interdisciplinar, possibilita oferecer apoio emocional para auxiliar os pais e as crianças no enfrentamento de situações referentes ao retorno ao ambiente familiar e contextos sociais.

Outro estado emocional identificado nos estudos foi a depressão, pois observou-se que os indicadores apresentaram variações ao longo do tempo decorrentes de fatores preditivos, por exemplo, a gravidade da queimadura, mas não exis-
Adriano Valério dos Santos Azevêdo e Maria Aparecida Crepaldi

tem relações diretas. Destacou-se que a depressão é proveniente de uma série de fatores, por exemplo, idade, personalidade, e para a análise dos sintomas é necessário considerar os múltiplos aspectos envolvidos.

Diante da compreensão das relações estabelecidas entre cuidador, criança e profissionais de saúde, é possível iniciar pesquisas que possibilitem o avanço no estudo de temáticas referentes às interações da tríade. A integração de familiares cuidadores nas pesquisas contribui para identificar pontos a serem investigados, por exemplo, investigar as relações estabelecidas no sistema familiar (Landolt, Ystrom, Sennhauser, Gnehm, \& Vollrath, 2012).

Constatou-se pelos resultados que a hospitalização decorrente de queimaduras provocou estresse parental e destacam-se as alterações emocionais e comportamentais da criança. Dessa forma, os pais apresentaram estresse em decorrência das reações da criança e do momento atual vivenciado por todos os integrantes da família, independentemente do tipo de lesão de queimadura. É importante salientar que os achados dos estudos enfatizam o humor e os comportamentos da criança, portanto é necessário compreender que os pais vivenciam culpa, o que se constata no trabalho empírico e clínico, e isso provoca estresse; e, fundamentalmente, à mãe que no imaginário social é quem deve zelar pelo bem-estar dos filhos.

Nessa perspectiva, iniciativas direcionadas para a construção de grupos de suporte para pais de crianças com queimaduras destacaram que a intervenção psicológica tem contribuído para os participantes relatarem suas dificuldades, e com isso auxiliar a utilização de estratégias de enfrentamento (Frenkel, 2008). Pesquisadores indicaram que a avaliação e intervenção psicológica direcionada para a criança com queimaduras apresenta repercussões na relação com o familiar cuidador, pois ambos desenvolvem e compartilham habilidades para o enfrentamento da hospitalização (Azevêdo, 2010; Azevêdo \& Santos, 2011). Por outro lado, a comunicação entre equipe de saúde, família e criança hospitalizada têm sido valorizada pelos profissionais de saúde que atuam em unidade de queimados (Lançoni-Júnior, Azevêdo, \& Crepaldi, 2017). Em síntese, a intervenção psicológica para familiares de crianças com queimaduras possibilita auxiliá-los nos aspectos que envolvem a relação com a criança; e o diálogo entre equipe de saúde, família e criança hospitalizada permite desenvolver vínculos afetivos, os quais contribuem para os familiares enfrentarem o período de hospitalização e de alta hospitalar, o que apresenta repercussões nas relações familiares.

Nesse sentido, no que se refere ao relacionamento familiar, os problemas familiares, ambientes propícios para abuso de crianças, e dificuldades financeiras foram aspectos identi- 


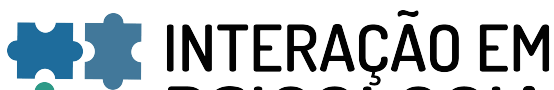 ET PSICOLOGIA}

ficados nas famílias de crianças com queimaduras. Um ponto a ser destacado se refere à maneira pela qual a família se organiza para a reinserção da criança na escola, é possível que alguns aspectos dificultem a readaptação escolar da criança, por exemplo, a imagem corporal, a aceitação dos colegas, o que representam implicações nas relações que são estabelecidas entre a criança, sua família e integrantes da escola. Os pais de crianças com queimaduras destacaram as dificuldades nas relações entre irmãos após o período de hospitalização, assim, faz-se necessário investigar o funcionamento dessas famílias. 0 tratamento requer dedicação dos pais e geralmente os irmãos não recebem a assistência integral, o que provoca ciúmes e rejeição. A aparência da criança que sofreu a queimadura e teve sequelas cicatriciais provoca uma sensação de choque nos irmãos, estes que precisam de um tempo para aceitar e adaptar-se à nova realidade familiar.

Existem fatores que influenciam na recuperação e reabilitação de queimaduras em crianças: o papel dos membros da família, o relacionamento familiar, reações dos pais, doenças psiquiátricas nos pais e doenças psiquiátricas pré-mórbidas nas crianças (De Souza, 2010). Compreender os múltiplos fatores envolvidos possibilita que os profissionais de saúde direcionem atenção à família para que seja possível investigar aspectos inerentes à dinâmica estabelecida entre os integrantes e, com isso, delimitar propostas de intervenção. Por exemplo, reações intensas de ansiedade dos pais repercutem nos comportamentos da criança e promovem baixa adesão ao tratamento devido ao medo dos procedimentos invasivos. A identificação desses estados emocionais permite orientar os pais com informações educativas sobre o manejo da ansiedade para que possibilite auxiliar a relação com a criança.

\section{CONSIDERAÇÕES FINAIS}

As pesquisas sobre cuidadores de crianças hospitalizadas com queimaduras estão concentradas em temáticas específicas: ansiedade e estresse pós-traumático, depressão, estresse parental e relacionamento familiar. Os estudos sobre estados emocionais foram predominantemente realizados por meio de método quantitativo, alguns utilizaram delineamento longitudinal para fins de acompanhar os pais num período de tempo. Esses estudos internacionais investigaram distintos objetos de estudo e apresentaram as limitações para fins de fomentar novas pesquisas, por exemplo, verificar as relações entre estados emocionais do familiar cuidador e tamanho da queimadura da criança. Infelizmente não foi possível identificar estudos que envolveram amostras do contexto brasileiro, portanto a investigação da temática referente ao familiar cuidador da criança com queimaduras está concentrada em países do contexto americano.

\section{Adriano Valério dos Santos Azevêdo e Maria Aparecida Crepaldi}

Destaca-se a necessidade de estudos sobre o funcionamento das famílias buscando analisar preditores de sintomas psicológicos e variáveis psicossociais. Recomenda-se a realização de pesquisas longitudinais para um acompanhamento adequado das variáveis de estudo e para comparar as vivências de pais no período de hospitalização e na fase de retorno ao contexto familiar. 0 apoio social aos pais e crianças representa outra temática para investigação nos próximos estudos.

A continuidade de pesquisas sobre estados emocionais (ansiedade, estresse, depressão) possibilita direcionar programas de intervenção psicológica e realizar acompanhamentos sistemáticos. Em síntese, os resultados dos estudos permitem inferir a importância dos serviços de saúde e de assistência social acompanharem os pais ao longo do tempo para oferecer suporte adequado às suas necessidades. Atenção psicológica nessa área permite integrar a família nas discussões referentes ao enfrentamento de situações, no período de hospitalização, no retorno gradual das atividades e no manejo nas relações familiares.

Diante das pesquisas que buscaram investigar estados emocionais por meio de instrumentos padronizados, recomenda-se que sejam realizados grupos de intervenção de maneira sistemática: verificar indicadores de ansiedade em familiares que são participantes de um grupo de relaxamento por meio de estudo experimental; e estudos qualitativos para investigar o relacionamento familiar após a alta hospitalar. Assim, espera-se que as pesquisas permitam apresentar resultados referentes às redes sociais significativas dos familiares cuidadores, e as relações entre estados emocionais e redes sociais, para a construção de programas e políticas públicas voltadas para a atenção de famílias de crianças com queimaduras durante e após o período de hospitalização.

\section{DECLARAÇÃO DA CONTRIBUIÇÃO DOS AUTORES}

A. V. S. A. realizou as buscas e escreveu os resultados provenientes dos estudos que foram selecionados. M. A. C. ficou responsável pela revisão do artigo e contribuiu no processo de organização das temáticas.

\section{DECLARAÇÃO DE CONFLITOS DE INTERESSE}

Os autores declaram que não há conflitos de interesse no manuscrito submetido. 


\section{W INERACÄOEM ET PSICOLOGIA}

\section{REFERÊNCIAS}

Azevêdo, A.V.S. (2010). Construção do protocolo de avaliação psicológica hospitalar para criança queimada. Avaliação Psicológica, 9(1), 99-109.

Azevêdo, A.V.S., \& Santos, A.F.T. (2011). Intervenção psicológica no acompanhamento hospitalar de uma criança queimada. Psicologia Ciência e Profissão, 31(2), 328-339. https://doi.org/10.1590/S1414-98932011000200010.

Bakker, A., Van Loey, N. E., Van Son, M.J., \& Van der Heijden, P.G. (2010). Brief report: mothers long-term posttraumatic stress symptoms following a burn event of their child. Journal of Pediatric Psychology, 35(6), 656-661. https:// doi.org/10.1093/jpepsy/jsp090.

Bakker, A., Van Loey, N.E.E., Van der Heijden, P.G.M., \& Van Son, M.J.M. (2012). Acute stress reactions in couples after a burn event to their young child. Journal of Pediatric Psychology, 37(10), 1127-1135. https://doi.org/10.1093/ jpepsy/jss083.

Bakker, A., \& Walstra, J. (2013). Psychological reactions on trauma in children and parents. In A. E. E. De Jong (Ed.), Burn care (pp. 145-154). Breda: Oxford

Bicho, D., \& Pires, A. (2002). Comportamento de mães de crianças hospitalizadas devido a queimaduras. Análise Psicológica, 1, 115-129.

Blakeney, P., Meyer, W., Moore, P., Murphy, L., Broemeling, L., Robson, M., \& Herndon, D. (1993a). Psychosocial sequelae of pediatric burns involving $80 \%$ or greater total body surface area. Journal of Burn Care \& Rehabilitation, 14(6), 684-689. https://doi.org/10.1016/0272-7358(91)90114-A

Blakeney, P., Moore, P., Broemeling, L., Hunt, R., Herndon, D. N., \& Robson, M. (1993b). Parental stress as a cause and effect of pediatric burn injury. Journal of Burn Care \& Rehabilitation, 14(1), 73-79. https://doi.org/ 10.1097/00004630-199301000-00016

Blakeney, P., Meyer, W., Robert, R., Desai, M., Wolf, S., \& Herndon, D. (1998). Long-term psychosocial adaptation of children who survive burns involving $80 \%$ or greater total body surface area. Journal of Trauma, 44(4), 625-632. https:// doi.org/10.1097/00005373-199707000-00109

De Souza, A. (2010). Psychological aspects of pediatrics burns (a clinical review). Annals of Burns and Fire Disasters, 23(3), 155-159.

El Hamaoui, Y., Yaalaoui, S., Chihabeddine, K., Boukind, E., \& Moussaoui, D. (2006). Depression in mothers of burned children. Archives of Women's Mental Health, 9(3), 117119. https://doi.org/10.1007/s00737-006-0124-1

Fukunishi, I. (1998). Posttraumatic stress symptoms and depression in mothers of children with severe burn injuries. Psychological Reports, 83(1), 331-335. https://doi.org/ 10.2466/PR0.83.5.331-335
Frenkel, L. (2008). A support group for parents of burned children: A South African Children's Hospital Burns Unit. Burns, 34(1), 565-569. https://doi.org/10.1016/j.burns. 2007.09.016

Gorga, D., Johnson, J., Bentley, A., Silverberg, R., Glassman, M., Madden, M., \& Nagler, W. (1999). The physical, functional, and developmental outcome of pediatric burn survivors from 1 to 12 months postinjury. Journal of Burn Care \& Rehabilitation, 20(2), 171-178.

Hall, E., Saxe, G., Stoddard, F., Kaplow, J., Koenen, K., Chawla, N., \& King, D. (2006). Posttraumatic stress symptoms in parents of children with acute burns. Journal of Pediatric Psychology, 31(4), 403-412. https://doi.org/10.1093/ jpepsy/jsj016

Horridge, G., Cohen, K., \& Gaskell, S. (2010). BurnEd: parental, psychological and social factors influencing a burn-injured child's return to education. Burns, 36(5), 630-638. https:// doi.org/10.1016/j.burns.2009.08.013

Joseph, K. E., Adams, C. D., Goldfarb, I. W., \& Slater, H. (2002). Parental correlates of unintentional burn injuries in infancy and early childhood. Burns, 28(5), 455-463. doi 10.1016/ S0305-4179(02)00035-9

Kazak, A. E., Kassam-Adams, N., Schneider, S., Zelikovsky, N., Alderfer, M.A., \& Rourke, M. (2006). An integrative model of pediatric medical traumatic stress. Journal of Pediatric Psychology, 31(4), 343-355. https://doi.org/10.1093/ jpepsy/jsj054

Kent, L., King, H., \& Cochrane, R. (2000). Maternal and child psychological sequelae in pediatric burn injuries. Burns, 26(4), 317-322.

Kilburn, N., \& Dheansa, B. (2014). Socioeconomic impact of children's burns- a pilot study. Burns, 40(8), 1615-1623. https://doi.org/10.1016/j.burns.2014.03.006

Lançoni-Júnior, A. C., Azevêdo, A. V. S., \& Crepaldi, M. A. (2017). Comunicação entre equipe de saúde, família, criança em unidade de queimados. Psicologia em Estudo, 22(4), 623-634. https://doi.org/colestud.v22i4.35849

Landolt, M.A., Grubenmann, S., \& Meuli, M. (2002). Family impact greatest: predictors of quality of life and psychological adjustment in pediatric burn survivors. Journal of Trauma, 53(6), 1146-1151. https://doi.org/10.1097/01.TA. 0000033763.65011 .89

Landolt, M. A., Buehlmann, C., Maag, T., \& Schiestl, C. (2009). Brief report: quality of life is impaired in pediatric burn survivors with posttraumatic stress disorder. Journal of Pediatric Psychology, 34(1), 14-21. https://doi.org/10.1093/ jpepsy/jsm088

Landolt, M. A., Ystrom, E., Sennhauser, F. H., Gnehm, H. E., \& Vollrath, M. E. (2012). The mutual prospective influence of child and parental post-traumatic stress symptoms in pediatric patients. Journal of Child Psychology and Psychiatry, 53(7), 767-774. https://doi.org/10.1111/j. 1469-7610.2011.02520.x 


\section{W INERAC̄̈OEM ET PSICOLOGIA}

LeDoux, J.M., Meyer, W. J., Blakeney, P. E., \& Herndon, D. N. (1998). Relationship between parental emotional states, family environment and the behavioral adjustment of pediatric burn survivors. Burns, 24(5), 425-432. https://doi.org/ 10.1016/S0305-4179(98)00038-2

Lehna, C. (2010). Sibling experiences after a major childhood burn injury. Pediatric Nursing, 36(5), 245-251.

Liber, J. M., List, D., Van Loey, N. E., \& Kef, S. (2006). Internalizing problem behavior and family environment of children with burns: A Dutch pilot study. Burns, 32(2), 165-171. https://doi.org/10.1016/j.burns.2005.10.008

Mancuso, M. G., Bishop, S., Blakeney, P., Robert, R., \& Gaa, J. (2003). Impact on the family: psychosocial adjustment of siblings of children who survive serious burns. Journal of Burn Care \& Rehabilitation, 24(2), 110-118. https://doi.org/ 10.1097/01.BCR.0000054169.02965.C4 ·

Martin-Herz, S. P., Thurber, C. A., \& Patterson, D. R. (2000) Psychological principles of burn wound pain in children. II: Treatment applications. Journal of Burn Care \& Rehabilitation, 21(5), 458- 472. https://doi.org/10.1046/j.15332500.2001.001002211.x

McGarry, S., Girdler, S., Mcdonaldo, A., Valentine, J., Wood, F., \& Elliot, C. (2013). Pediatric medical trauma: The impact on parents of burn survivors. Burns, 39(6), 1114-1121. https://doi.org/10.1016/j.burns.2013.01.009

McGarry, S., Eliott, C., McDonald, A.,Valentine, J., Wood, F., \& Girdler, S. (2015). "This is not just a little accident": a qualitative understanding of pediatric burns from the perspective of parents. Disability Rehabilitation, 37(1), 41-50. https:// doi.org/10.3109/09638288.2014.892640

Mendes, K.D.S., Silveira, R.C.C.P., \& Galvão, C.M. (2008). Revisão integrativa: Método de pesquisa para a incorporação de evidências na saúde e na Enfermagem. Texto Contexto Enfermagem, 17(4), 758-764. https://doi.org/10.1590/ S0104-07072008000400018

Meyer, W. J., Blakeney, P., Moore, P., Murphy, L., Robson, M., \& Herndon, D. (1994). Parental wellbeing and behavioral adjustment of pediatric survivors of burns. Journal of Burn Care \& Rehabilitation, 15(1), 62-68. https://doi.org/ 10.1097/00004630-199401000-00012

Meyer, W. J., Robert, R., Murphy, L., \& Blakeney, P. E. (2000). Evaluating the psychosocial adjustment of 2- and 3-yearold pediatric burn survivors. Journal of Burn Care \& Rehabilitation, 21(2), 179-184. https://doi.org/ 10.1097/00004630-200021020-00019

Nelson, L.P., \& Gold, J.I. (2012). Posttraumatic stress disorder in children and their parents following admission to the pediatric intensive care unit: a review. Pediatric Critical Care Medical, 13(3), 338-347. https://doi.org/10.1097/PCC Ob013e3182196a8f
Adriano Valério dos Santos Azevêdo e Maria Aparecida Crepaldi

Oliveira, V.V., Fonseca, A.S., Leite, M.T.S., \& Santos, L.S. (2015). Vivência dos pais no enfrentamento da situação de queimaduras em um filho. Revista Rene, 16(2), 201209.

https://doi.org/10.15253/21756783.2015000200009

Oster, C., Hensing, I., Lojdstrom, T., Sjoberg, F., \& Willebrand, M. (2014). Parents perceptions of adaptation and family life after burn injuries in children. Journal of Pediatric Nursing, 29(6), 606-613. https://doi.org/10.1016/j.pedn. 2014.06.010

Pardo, G. D., Garcia, I. M., Marrero, F.R.M., \& Cia, T.G. (2008). Psychological impact of burns on children treated in a severe burns unit. Burns, 34(7), 986-993. https://doi.org/ 10.1016/j.burns.2008.01.016

Peck, M. D., \& Priolo-Kapel, D. (2002). Child abuse by burning: a review of the literature and an algorithm for medical investigations. Journal of Trauma, 53(5), 1013-1022. https:// doi.org/10.1097/01.TA.0000023163.78766.FF

Phillips, C., Fussell, A., \& Rumsey, N. (2007). Considerations for psychosocial support following burn injury: a family perspective. Burns, 33(8), 986-994. https://doi.org/ 10.1016/j.burns.2007.01.010

Phillips, C., \& Rumsey, N. (2008). Considerations for the provision of psychosocial services for families following pediatric burn injury - a quantitative study. Burns, 34(1), 56-62. https://doi.org/10.1016/j.burns.2006.12.003

Rivlin, E., \& Faragher, E.B. (2007). The psychological sequelae on mothers of thermally injured children and adolescents: future directions: Part 3. Developmental Neurorehabilitation, 10(2), 183-190. https://doi.org/ $10.1080 / 17518420701309758$

Rizzone, L.P., Stoddard, F.J., Murphy, J. M., \& Kruger, L.J. (1994). Posttraumatic stress disorder in mothers of children and adolescents with burns. Journal of Burn Care \& Rehabilitation, 15(2), 158-163. https://doi.org/ 10.1097/00004630-199403000-00010

Rosenberg, L., Blakeney, P., Thomas, C. R., Holzer, C. E., Robert, R. S., \& Meyer, W. J. (2007). The importance of family environment for young adults burned during childhood. Burns, 33(5), 541-546. https://doi.org/10.1016/j.burns. 2006.11.005

Sheridan, R. L. (2002). Burns. Critical Care Medicine, 30(11), 500-514.

Suurmond, J.L., Van Loey, N., Dokter, J., \& Essink-Bot, M. L. (2011). Coping with guilt: coping strategies of ethnic minority and Dutch parents of children with pediatric burns. Burns, 37(1), 3-4. https://doi.org/10.1016/S03054179(11)70015-8 


\section{-4: INTERACÃO EM LF PSICOLOGIA}

Thombs, B. D. (2008). Patient and injury characteristics, mortality risk, and length of stay related to child abuse by burning: evidence from a national sample of 15,802 pediatric admissions. Annals of Surgery, 247(3), 519-523. https:// doi.org/10.1097/SLA.0b013e31815b4480

Thompson, R., Boyle, D., Teel, C., Wambach, K., \& Cramer, A. (1999). A qualitative analysis of family member needs and concerns in the population of patients with burns. Journal of Burn Care \& Rehabilitation, 20(6), 487-496. https:// doi.org/10.1097/00004630-199920060-00011

Vendrusculo, T.M., Balieiro, C.R.B., Echevarría-Guanilo, M.E., Farina-Junior, J.A., \& Rossi, L.A. (2010). Queimaduras em ambiente doméstico: características e circunstâncias do acidente. Revista Latino Americana de Enfermagem, 18(3), 444-451. https://doi.org/10.1590/S0104-

\section{Adriano Valério dos Santos Azevêdo e Maria Aparecida Crepaldi}

Wibbenmeyer, L., Lião, J., Heard, J., Kealey, L., Kealey, G., \& Oral, R. (2014). Factors related to child maltreatment in children presenting with burn injuries. Journal of Burn Care \& Research, 35(5), 374-381. https://doi.org/10.1097/BCR. 0000000000000005

11692010000300021 